\title{
Studying properties of neurotransmitter receptors by non-stationary noise analysis of spontaneous postsynaptic currents and agonist-evoked responses in outside-out patches
}

\author{
Espen Hartveit \& Margaret Lin Veruki \\ Department of Biomedicine, University of Bergen, Jonas Lies vei 91, N-5009 Bergen, Norway. Correspondence should be addressed to E.H. \\ (espen.hartveit@biomed.uib.no).
}

Published online 8 March 2007; doi:10.1038/nprot.2007.47

\begin{abstract}
Chemical synaptic transmission depends on neurotransmitter-gated ion channels concentrated in the postsynaptic membrane of specialized synaptic contacts. The functional characteristics of these neurotransmitter receptor channels are important for determining the properties of synaptic transmission. Whole-cell recording of postsynaptic currents (PSCs) and outside-out patch recording of transmitter-evoked currents are important tools for estimating the single-channel conductance and the number of receptors contributing to the PSC activated by a single transmitter quantum. When single-channel activity cannot be directly resolved, non-stationary noise analysis is a valuable tool for determining these parameters. Peak-scaled non-stationary noise analysis can be used to compensate for quantal variability in synaptic currents. Here, we present detailed protocols for conventional and peak-scaled non-stationary noise analysis of spontaneous PSCs and responses in outside-out patches. In addition, we include examples of computer code for individual functions used in the different stages of non-stationary noise analysis. These analysis procedures require $3-8 \mathrm{~h}$.
\end{abstract}

\section{INTRODUCTION}

Chemical synaptic transmission

Chemical synaptic transmission is an important mode of communication between neurons ${ }^{1}$. Both excitatory and inhibitory chemical synaptic transmission depend on transmitter-gated ion channels that are concentrated in the postsynaptic membrane of specialized synaptic contacts. To understand the functional properties of a specific synapse, it is important to understand the functional characteristics of the transmitter-gated ion channels involved. These characteristics include single-channel conductance (Box 1) and kinetics, the number and density of receptors in the postsynaptic membrane and the average number of ion channels open at the peak of a postsynaptic current (PSC) (see review by Silver and Farrant $^{2}$ ). Many synapses are located in the dendrites of neurons, far from the soma, where a recording electrode is typically located. This makes it difficult to investigate the receptor channels responsible for mediating synaptic transmission. In addition to the inaccessibility of synaptic ion channel receptors for direct recording, the physical and/ or electrical remoteness of synapses introduces problems of electrotonic cable filtering that will distort the signal recorded at the soma relative to the signal generated locally at the synapse ${ }^{3-6}$.

For electrophysiological investigations of synaptic transmittergated ion channels, one would ideally like to achieve two different recording configurations. First, one would like to perform whole-cell voltage-clamp (Box 1) recordings as close to the synapse as possible to optimize the degree of voltage-clamp. Second, one would like to isolate outside-out membrane patches (Box $\mathbf{1})$ as close to the synapse as possible to minimize potential problems of functional differences between synaptic and extrasynaptic receptors. Except in a few cases, either goal is very difficult to achieve. With respect to voltage-clamp control, dendritic and axon terminal recordings have made it possible to improve the degree of space clamp (Box 1) of synaptic currents (e.g., see refs. 6-11), but with the obvious limitation that the control is still spatially restricted and synaptic inputs generated elsewhere in the dendritic tree are less likely well voltage-clamped owing to generally higher electrode series resistance in dendritic as opposed to somatic recordings. With respect to outside-out patches, they can be used for high-resolution recording of either singlechannel activity or macroscopic responses where the channels are activated by ultrafast application of receptor agonists ${ }^{12}$ to mimic the temporal concentration profile of neurotransmitter in a chemical synapse $^{13}$. Although initial investigations focused on somatic patches (e.g., see refs. 14-17), it has, in some cases, also been possible to isolate dendritic or axon terminal patches (e.g., see refs. 10,11,18-20).

The PSC caused by release of a single transmitter vesicle is termed a quantal or miniature PSC (mPSC ${ }^{21,22}$; Box 1). In preparations where evoked release is caused by presynaptic action potentials involving voltage-gated $\mathrm{Na}^{+}$channels, recording mPSCs requires the blocking of presynaptic action potentials with tetrodotoxin (TTX). However, not all synapses depend on presynaptic, TTX-sensitive action potentials and some spontaneous PSCs (spPSCs) might be caused by synchronous, multivesicular release. Thus, in this protocol we use the term spPSCs to represent spontaneous, action potential-independent PSCs. One approach to estimating the number of ion channels open at the peak of an spPSC is to divide its amplitude by the single-channel (unitary) current. The single-channel current can be obtained from outsideout patches, either by directly resolved channel activity or by conventional non-stationary noise analysis (described below). In some cases, it is also possible to resolve single-channel activity directly in the later phases of the decay of spPSCs. The likelihood of obtaining the necessary high-resolution recordings increases when the ion channels involved have a large single-channel conductance 


\section{BOX 1 | GLOSSARY}

1. AMPA receptors: non-selective cation receptor channels, subtype of ionotropic glutamate receptors, defined by activation by the synthetic agonist $\alpha$-amino-3-hydroxy-5-methyl-4-isoxazolepropionic acid (AMPA). Involved in fast synaptic excitation in several CNS synapses.

2. $\mathrm{GABA}_{\mathrm{A}}$ receptors: chloride-selective receptor channels activated by the neurotransmitter $\gamma$-aminobutyric acid (GABA), blocked by the competitive antagonist bicuculline. Responsible for the majority of fast synaptic inhibition in the CNS.

3. Glycine receptors: chloride-selective receptor channels activated by the neurotransmitter glycine. Involved in fast synaptic inhibition in the brain stem, spinal cord and retina.

4. Ionotropic receptor: receptor channel, i.e., a receptor that contains an ion channel as an integral part of the macromolecular complex.

5. NMDA receptors: non-selective cation receptor channels, subtype of ionotropic glutamate receptors, defined by activation by the synthetic agonist $\mathrm{N}$-methyl-D-aspartic acid (NMDA). Involved in fast synaptic excitation in several CNS synapses.

6. Outside-out patch recording: recording configuration of the electrophysiological patch-clamp technique whereby a piece of cell membrane has been excised from a cell in such a way that the outward-facing side of the membrane is exposed to the extracellular solution.

7. Quantal variability: variability of the quantal size (amplitude). Can appear as variability in the response to repeated release of a single vesicle of transmitter from a single release site or as variability in the responses to release from the different release sites encompassed by a single synaptic connection.

8. Quantum (quantal postsynaptic current): the elementary unit of synaptic transmission and the smallest possible postsynaptic response. A given postsynaptic response is built up from one or more quanta. In physical terms, considered to be the postsynaptic response activated by transmitter released from a single vesicle.

9. Release site: the specific location in a chemical synapse where transmitter vesicles are released from the presynaptic neuron.

10. Series resistance: the electrical resistance between the interior of the tip of a patch pipette and the interior of a cell during a whole-cell recording. When current flows, the series resistance is responsible for a corresponding voltage drop. The series resistance will typically be higher (but never lower) than the resistance of the patch pipette used in the recording.

11. Single-channel conductance: the electrical conductance ( $G$; inverse of resistance, $R$ ) of a single ion channel. Denoted by $\gamma$ (measured in Siemens; $S)$; typical single-channel conductances are in the picoSiemens (pS) range.

12. Space clamp: the spatial extent to which a neuron is under adequate control of a voltage clamp. For example, when a voltage clamp is imposed by an electrode at the soma of a neuron, the voltage clamp of the dendrites will typically be less effective.

13. Voltage clamp: an electrophysiological recording technique where an electronic circuit is used to control (clamp) the membrane potential of a cell by keeping it constant at a given value. If current flows through ion channels in the cell membrane, the electronic circuit injects current of the same magnitude and opposite polarity to keep the membrane potential constant.

14. Whole-cell recording: recording configuration of the electrophysiological patch-clamp technique whereby the tip of a blunt, low-resistance (typically 1-10 M $\Omega$ ) glass pipette is tightly sealed to the cell membrane (resistance $\geq 1-10 \mathrm{G} \Omega$ ), followed by breaking the area of cell membrane (patch) within the lumen of the tip, thus gaining electrical access to the interior of the cell.

and open state(s) of long duration and when the synaptic inputs are located close to the recording electrode. Directly resolved single-channel activity in PSCs has been observed for glycine receptors $^{23-26}, N$-methyl-D-aspartic acid (NMDA) receptors ${ }^{27-30}$ and $\gamma$-aminobutyric acid type $\mathrm{A}\left(\mathrm{GABA}_{\mathrm{A}}\right)$ receptors $^{31}$ (Box 1). It has not been possible to obtain directly resolved single-channel openings in spPSCs mediated by $\alpha$-amino-3-hydroxy-5-methyl-4isoxazolepropionic acid (AMPA) receptor channels, which often have open states with very short durations (for review, see ref. 32). Against this background, we discuss options and techniques available for investigating the single-channel conductance and the number of transmitter-gated ion channels involved in mediating chemical synaptic transmission by non-stationary noise analysis. The focus is on analysis of spPSCs and responses from outside-out patches.

\section{Conventional non-stationary noise analysis}

Before the development of single-channel recording techniques ${ }^{33,34}$, noise analysis (alternatively termed fluctuation analysis) was the only available method that could yield information on singlechannel parameters. Noise analysis is performed by recording the current fluctuations associated with stochastic gating among a population of ion channels. Although single-channel recording is inherently a much more powerful technique than noise analysis, there are still situations where noise analysis techniques can offer valuable information not easily obtained otherwise. For example, for ion channels with very low single-channel conductance, the resolution of single-channel recording can be insufficient to resolve the gating activity. In these cases, noise analysis can be used to yield information on the single-channel parameters. Noise analysis was developed for stationary conditions in which the stimulus amplitude and average ion channel activity are constant over time (for reviews, see refs. 35-37). If the ion channels of interest rapidly inactivate or desensitize to a very low steady-state response amplitude, and/or the stimulus is inherently transient, stationary noise analysis cannot be applied. To compensate for this, noise analysis was extended to handle non-stationary conditions ${ }^{38-40}$. Thus, for what is referred to as conventional non-stationary noise analysis, an invariant population of ion channels is repeatedly subjected to a constant stimulus. Examples of experimental approaches where this type of analysis is appropriate are agonist-evoked responses in outside-out patches (see above), two-photon uncaging of caged glutamate onto dendritic spines ${ }^{41}$ and spPSCs originating from a single release site $^{42}$. It has also been argued that conventional nonstationary noise analysis can be appropriate for analysis of spPSCs mediated by NMDA receptors because high intrinsic variance in the time from binding of agonist to the first opening introduces considerable variability with respect to the location of the peak, thus complicating the use of peak scaling (see below; ref. 43).

From the ensemble of responses, one calculates the mean current response and the variance of current fluctuations about the mean 
for each point in time. The average current for an ensemble of $n$ events, where each event contains $m$ sample points, is given by the following equation (e.g., ref. 44):

$$
\operatorname{Avg}(i)=\frac{1}{n} \sum_{j=1}^{n} y(i)_{j}, \quad i=1, \ldots, m,
$$

where $i$ is the index of the sample point $(1, \ldots, m)$ and $j$ is the index of the event $(1, \ldots, n)$. With regular sampling (i.e., constant sampling interval of $\Delta t$ ), the index $i$ of the sample points will correspond to time points $i \times \Delta t$ (or $(i-1) \times \Delta t$ if the first point in the array is considered to correspond to time zero). The ensemble variance about each sample point $(i)$ in the ensemble mean is given by the following equation:

$$
\operatorname{Var}(i)=\frac{1}{n-1} \sum_{j=1}^{n}\left[y(i)_{j}-\operatorname{Avg}(i)\right]^{2}, \quad i=1, \ldots, m
$$

The result of these calculations is that a separate mean and variance is calculated for each point in time. A plot of corresponding values of variance versus mean, for each point in time, can then be fitted with the following equation ${ }^{38}$ :

$$
\sigma^{2}(I)=i I-\frac{I^{2}}{N}+\sigma_{\mathrm{b}}^{2}
$$

developed from a binomial model ${ }^{37}$, yielding values for single-channel current $(i)$ and total number of ion channels available for activation $\left(\mathrm{N} ; \sigma_{\mathrm{b}}^{2}\right.$ is the background variance). The single-channel (unitary) chord conductance $(\gamma)$ can then be calculated as:

$$
\gamma=i /\left(V_{\mathrm{m}}-E_{\text {rev }}\right)
$$

from the known membrane holding potential $\left(V_{\mathrm{m}}\right)$ and reversal potential $\left(E_{\text {rev }}\right)$. The peak open probability $\left(P_{\mathrm{o} \text {, peak }}\right)$, corresponding to the fraction of available ion channels open at the time of the peak current $\left(I_{\text {peak }}\right)$, can then be calculated from the following equation:

$$
P_{\mathrm{o}, \text { peak }}=I_{\text {peak }} / N i
$$

\section{Peak-scaled non-stationary noise analysis}

As discussed above, conventional non-stationary noise analysis can be applied only to ensembles of time-varying responses for which the population of contributing ion channels is invariant from one response to the next (i.e., the current variance arises solely from stochastic ion channel gating and time-invariant background noise). However, if there is variation in the number of release sites (as for repeatedly evoked PSCs) and/or variation in the identity of release sites (as for spPSCs), the application of conventional non-stationary noise analysis is problematic ${ }^{45,46}$ (for reviews, see refs. 2,47). In these cases, quantal variability (both quantal size and number of quanta; Box 1) will contribute to the variability in PSC amplitude. It, therefore, becomes necessary to isolate variations in currents arising from the stochastic gating of ion channels from variations arising from sources such as quantal variability and variability of release sites. For conventional non-stationary noise analysis, fluctuations around the mean are isolated by directly subtracting the mean response waveform from each individual response waveform (see above). Thus, for analysis of PSCs arising from more than one release site (evoked PSCs or spPSCs), scaling the mean PSC waveform to the peak amplitude of each individual PSC waveform before subtracting them from each other should isolate fluctuations around the mean arising from stochastic ion channel gating ${ }^{45,48}$ (for review, see ref. 2). An important consequence of this peak-scaling method is that information on the total number of available channels - that is, the number of channels exposed to neurotransmitter $(N)$-is lost (see below). Above all, this peak-scaled non-stationary noise analysis is applicable to spPSCs. An advantage of spPSCs, compared with evoked PSCs, is that with spPSCs, there should be minimal asynchrony in the activation of receptor channels. However, by necessity, the number and identity of channels activated will change from one spPSC to the next. As summarized in a recent review ${ }^{2}$, a series of assumptions must be fulfilled for peak-scaled non-stationary noise analysis of spPSCs to be valid. First, the contributing receptor channels have to be identical. If they are not, the resulting estimate for $i$ will be a weighted estimate of a mean channel conductance ${ }^{49}$. Second, the contributing channels have to be independent. For example, if they can influence each other by compromising the effectiveness of the voltage clamp, this condition will not be fulfilled. Third, the mean PSC waveform should be the same at all contributing synapses. This means that the synapses contributing to the ensemble of recorded spPSCs must have the same (or similar) electrotonic location. It is, therefore, important to test whether the ensemble of recorded events display stability over time (within a given experimental condition) and whether there is evidence for significant, differential electrotonic filtering of the events ${ }^{5,22}$. Finally, although conventional non-stationary noise analysis applied to an invariant population of ion channels always gives rise to a parabolic variance versus mean relationship, peak-scaled non-stationary noise analysis can give rise to either a parabolic or a non-parabolic (skewed) variance versus mean relationship. In cases where the peak-scaled variance versus mean relationship is parabolic, the estimate for $N$, obtained from curve fitting with equation (3), will correspond approximately to the average number of channels open at the peak of the PSC ${ }^{45,46}$, but in cases where the peak-scaled variance versus mean relationship is skewed, it can be only partially fitted with equation (3) and the estimate for $N$ will have no direct physical interpretation (see ref. 50 for an extensive analysis). The shape of the variance versus mean relationship obtained by peak scaling (parabolic versus skewed) does not depend on whether the underlying population of ion channels is invariant. Rather, the degree of skewness depends on the temporal structure of the fluctuations around the mean during the decay phase after the peak response and can be investigated with covariance analysis of the ensemble of $\mathrm{PSCs}^{50}$ (see below). Skewed variance versus mean relationships have been observed both for physiologically recorded PSCs and for simulated responses based on Markov-style kinetic schemes ${ }^{45,50-53}$.

\section{Autocovariance analysis}

The autocovariance (for simplicity, termed covariance) function in the time domain is equivalent to the power spectral density function in the frequency domain (e.g., ref. 54) and indicates how well the fluctuations are correlated at different times ${ }^{39,40}$. Using covariance analysis as a tool, it has been demonstrated that peak scaling generates a skewed variance versus mean relationship when the covariance function decays rapidly (compared with the response waveform of the ensemble mean), corresponding to a low correlation between fluctuations at the peak and in neighboring regions of the decay phase ${ }^{50}$. A parabolic relationship is obtained when the covariance function decays more slowly, corresponding to 
a higher correlation. For a response ensemble with $n$ events, each with $m$ sample points, the covariance function can be calculated from the corresponding $m \times n$ event matrix. The resulting $m \times m$ covariance matrix is a measure of the linear strength between the $m$ variables and can be stated formally as

$$
\begin{gathered}
C(i, j)=\frac{1}{n-1} \sum_{k=1}^{n}\left[y_{k}(i)-\operatorname{Avg}(i)\right]\left[y_{k}(j)-\operatorname{Avg}(j)\right], \\
i=1, \ldots, m, \quad j=1, \ldots, m,
\end{gathered}
$$

where the $y_{k}$ are the current values from the $k$ th event and the Avg are the means of the $n y_{k}$ values at samples $i$ and $j$ (refs. 39,40). $\mathrm{C}(i, j)$ is a $2 \mathrm{D}$ function that represents the correlation between the variances at samples $i$ and $j$ within the record. Each value in the covariance matrix, $\sigma_{i j}{ }^{2}$, corresponds to the covariance between the corresponding rows $i$ and $j$ in the original event matrix. The diagonal of the covariance matrix (for $i=j$ ) corresponds to the ensemble variance, that is, the $m$ variance values across the $n$ columns of the event matrix. For a given center point (at $i$ or $t_{\mathrm{c}}$ ), the decay of a covariance function, $\mathrm{C}(i, j)$, can be estimated by fitting with exponential functions such that each decay time constant corresponds to a correlation time ( $\left.t_{\text {corr }}\right)$. Depending on the number of sample points $(m)$ and the number of events $(n)$, calculation of $\mathrm{C}(i, j)$ can be computationally demanding. Although $\mathrm{C}(i, j)$ contains considerable kinetic information, this type of analysis has mainly been used for voltage-gated ion channels ${ }^{39,40}$, and less for neurotransmitter receptor channels (e.g., see ref. 55).

A potential application of covariance analysis arises from the need to evaluate the shape of a variance versus mean relationship obtained by peak-scaled non-stationary noise analysis of spPSCs. Although a skewed, as opposed to parabolic, relationship can be caused by, for example, inadequate alignment of spPSCs, it can also be caused by the kinetic properties of the receptor channels themselves ${ }^{50}$. If it is possible to perform additional experiments with application of agonist to outside-out patches (with receptor channels similar to the synaptic ones), the results can be analyzed using peak-scaled, in addition to conventional, non-stationary noise analysis. If peak-scaling generates a skewed variance versus mean relationship, it can be assumed that this is caused by the kinetic properties of the receptor channels and that this is also the cause of a skewed variance versus mean relationship obtained from peak-scaled non-stationary noise analysis of spPSCs from the same cell type. This hypothesis can be further corroborated by computing the covariance function from the ensemble of patch responses. A comparison of the decay of the macroscopic patch current with the decay of the covariance function (with center point corresponding to the peak amplitude of the macroscopic current) can indicate whether a skewed or parabolic variance versus mean relationship should be expected ${ }^{50}$.

\section{Bootstrap analysis}

Statistical errors in the best-fit parameters obtained by nonstationary noise analysis can be estimated by bootstrap analysis ${ }^{56}$ of the ensemble of events. Balanced resampling for a given ensemble with $n$ events can be achieved by generating, for example, 100 lists, each with $n$ randomly selected event numbers. Each number should correspond to an event in the original ensemble, and each list of numbers can be used to generate a synthetic, resampled data set with $n$ events. Examples of bootstrap analysis to estimate statistical errors in non-stationary noise analysis of experimental and simulated data can be found in refs. 50,52.

\section{MATERIALS}

EQUIPMENT

- Patch-clamp recording setup (e.g., see refs. 57-61)

-Equipment for ultra-fast application of agonists to outside-out membrane patches $^{12}$

- Software for detection of spPSCs (see EQUIPMENT SETUP)

- Software for noise analysis (see EQUIPMENT SETUP)

\section{EQUIPMENT SETUP}

Patch-clamp recording setup Detailed descriptions of equipment for patchclamp recording experiments and procedures for patch-clamp electrophysiology can be found in refs. 57-61. Experiments can be performed in vitro with cell cultures or slices or in vivo with whole animals. Both in vitro and in vivo recordings can be used to obtain recordings with evoked and/or spontaneous synaptic currents. The review by Trussell ${ }^{22}$ contains detailed descriptions of recording and analysis of synaptic currents. Experiments where one aims to obtain responses evoked by ultrafast agonist application to outside-out patches are ideally performed with cell culture or slice preparations (see ref. 12 for a detailed overview of the technique of ultrafast application to outside-out patches).

Software for detection of spPSCs Some packages (commercial or free) for computerized acquisition and/or analysis of patch-clamp electrophysiological data contain software for the automatic detection of spontaneous synaptic currents. Some examples are pClamp from Molecular Devices (http:// www.moleculardevices.com), AxoGraph X from AxoGraph Scientific (http://www.axographx.com), WinEDR/WinWCP in the Strathclyde Electrophysiology Software (http://spider.science.strath.ac.uk/sipbs/ showPage.php?pageName=software_ses), NeuroMatic from ThinkRandom by Jason Rothman (http://www.neuromatic.thinkrandom.com; requires the IGOR Pro software package; see below) and Patcher's Power Tools from the Max PlanckInstitute for Membrane Biophysics (http://www.mpibpc.gwdg.de/abteilungen/ 140/software/index.html; requires the IGOR Pro software package; see below).
In addition, the stand-alone software program Mini Analysis from Synaptosoft (http://www.synaptosoft.com) is often used for detecting spontaneous events. Software for noise analysis Although some software packages for computerized data acquisition and analysis contain functions that can be used for certain aspects of non-stationary noise analysis, many users will eventually prefer to develop their own software. This can be done in a general-purpose environment for software development, with the option to add scripting to the analysis. These environments offer many high-level functions and will, for most users, represent a convenient compromise between speed of execution and the ability to accomplish complex programming tasks in less time with minimal effort. Several packages are available, including IGOR Pro from Wavemetrics (http://www.wavemetrics.com), MATLAB from The Mathworks (http:// www.mathworks.com), Mathematica from Wolfram Research (http:// www.wolfram.com) and LabVIEW from National Instruments (http:// www.ni.com). GNU Octave (http://www.octave.org) has a syntax that is similar to that of MATLAB, but comes without a graphical user interface. In addition, AxoGraph X (see above), although geared toward analysis of electrophysiological data, offers many programmable functions in a general-purpose environment. For the analysis procedures used in this protocol, we have included some examples of computer code developed in the IGOR Pro environment (Supplementary Data). The code examples focus on the essential components of non-stationary noise analysis. For complete functionality, additional code for data input, data plotting, curve fitting and so on is required.

The Mini Analysis software package also offers functionality for non-stationary noise analysis. It is very important to validate the results with simulated data before applying this or similar software (supplied without available source code) to experimental data. A freely available, but specialized, software package for non-stationary noise analysis is NSFA by Tim Benke (http://www.bris.ac.uk/depts/Synaptic/research/software/NSFA_TCABLE/ NSFA_TCABLE_intro.htm; source code included). 


\section{PROCEDURE}

Data acquisition TIMING Approximately 6-12 $\mathrm{h}$ for each recording session (depending on experimental design) 1) Make sure that data are acquired at a sufficiently high sampling (digitization) frequency and with appropriate low-pass filtering. According to the Nyquist theorem, the minimum valid sampling frequency is two times the highest frequency component present in the analog signal to avoid aliasing (see discussion in ref. 44). In practice, the minimum valid sampling frequency is taken as two times the cut-off frequency of the low-pass filter. The faster the rise and decay times of the spPSCs are, the higher the sampling frequency must be. With the huge data-storage capacity of hard drives in modern computers, a safe strategy is to acquire data with a sampling frequency of $100 \mathrm{kHz}$ (corresponding to a sampling interval of $10 \mu \mathrm{s}$ ) at a high recording bandwidth (low-pass filter set to, e.g., 5 or $10 \mathrm{kHz}$ ) and to decimate (down-sample) and refilter data records as needed for off-line analysis. During acquisition, it is also necessary to make sure that data are sampled at an adequate resolution. As the amplitudes of the stochastic fluctuations can be small compared with the amplitudes of the transient current responses, it is advisable to employ an A/D converter with 16-bit, rather than 12-bit, resolution ${ }^{44}$ and to amplify the signal sufficiently before digitization to take advantage of the high amplitude resolution.

$\Delta$ CRITICAL STEP High quality, low-noise patch-clamp recordings are of invaluable importance for the following off-line analysis.

2| Depending on the goals of the project and the specific questions being asked, non-stationary noise analysis can be applied to data in the form of (i) spPSCs from continuous recordings (Fig. 1a) or (ii) responses evoked by ultrafast agonist application to outside-out patches. Depending on the project, it can be desirable to obtain both types of experimental data from a given cell type (see ANTICIPATED RESULTS).

\section{(A) Acquiring data with spPSCs from whole-cell recordings (continuous sampling)}

(i) Given the huge data-storage capacity of hard drives in modern computers, the electrical activity of the recorded cell should be sampled continuously (gap-free), with no need to perform trigger-activated sampling in response to on-line detection of spPSCs.

(ii) During data acquisition, it is very important to monitor the series resistance (access resistance; Box 1) of the recording. Although the series resistance should be as low as possible to minimize low-pass filtering of recorded events $6,53,62$ (see ref. 50 for detailed examples of distortion of single-channel conductance estimates from non-stationary noise analysis), changes in the series resistance can also compromise the results. One way to monitor series resistance is to design your sampling protocol in such a way that it interleaves continuous data acquisition (e.g., for 30-60 s) with a sequence that repeatedly samples the capacitative charging transients evoked by a square-wave stimulus (after canceling the cell capacitance neutralization circuitry of the patch-clamp amplifier). After averaging, the response can be used to calculate the series resistance value during off-line analysis (see below). In addition, during data acquisition, direct attention to the peak amplitude of capacitative charging transients can give valuable on-line information on the series resistance and changes in this important parameter.

(B) Acquiring data with responses evoked by ultrafast agonist application to outside-out patches (epochal sampling)

(i) Data should be acquired in epochs, with each epoch (sweep) corresponding to a single, stimulus-synchronized, response. In this way, there is no need to perform off-line detection of responses before further analysis. For non-stationary noise

Figure 1 | Properties and analysis of spontaneous postsynaptic currents (spPSCs). (a) Example of spPSCs in a whole-cell recording from an AII amacrine cell in an in vitro slice preparation of rat retina (maintained at room temperature; $20-24^{\circ}$ ). The spPSCs are glycinergic, inhibitory spPSCs (spIPSCs) recorded at a holding potential of $-60 \mathrm{mV}$ with a chloride equilibrium potential of approximately $0 \mathrm{mV}$ and appear as transient inward currents with fast rise times and slower decays. (b) Four examples of overlaid spIPSCs (as in panel a), aligned by the point of steepest rise. A single-exponential fit (red trace) has been overlaid on each spIPSC. (c) Average waveform of an ensemble of spIPSCs illustrates quantification of waveform parameters (indicated by vertical and horizontal lines): peak amplitude (amplitude interval between baseline and peak response), $10-90 \%$ rise time (time interval between the 10 and $90 \%$ amplitude values of the rising phase), half-decay time (time interval between the peak amplitude and the 50\% amplitude value of the decay phase) and time constant of decay ( $\tau_{\text {decay; }}$ single-exponential fit indicated by overlaid red trace). (d) Distribution of peak amplitude for an ensemble of spIPSCs (as in panel a); bin width $2.5 \mathrm{pA}$. The noise distribution is shown as an unfilled histogram with the peak scaled to the peak of the spIPSC amplitude distribution. Data from the same AII amacrine cell in $\mathbf{d}-\mathbf{f}$. (e) Distribution of $10-90 \%$ rise time for spIPSCs; bin width $0.025 \mathrm{~ms}$. (f) Distribution of $\tau_{\text {decay }}$ (single-exponential fit) for spIPSCs; bin width 0.25 ms. Data in panels b-f modified, with permission, from ref. 26, (C) (2006) The Physiological Society.

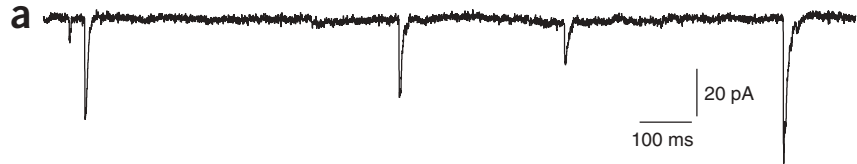

b
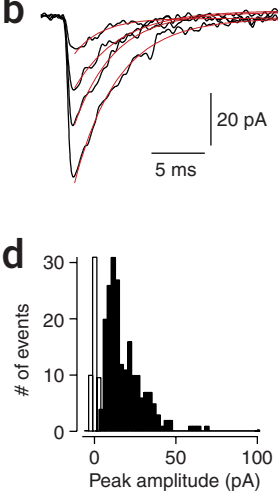

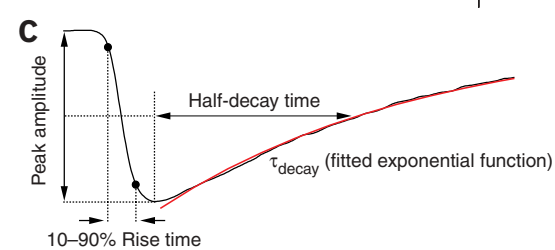

e
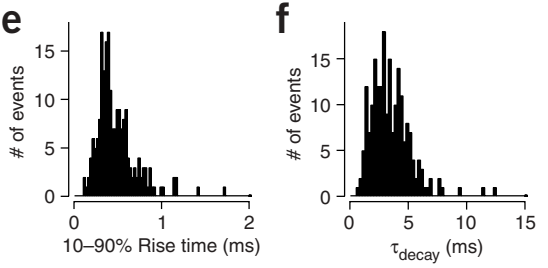
analysis, it is generally sufficient to acquire 50-100 consecutive responses. You should ensure that the sweep interval (the time interval between consecutive stimulus applications) is long enough that the data are not influenced by desensitization processes with incomplete recovery between sweeps. Depending on the channels studied, it may be necessary to use intervals up to, say, $15 \mathrm{~s}$.

PAUSE POINT Data can be analyzed any time after recording.

\section{Detect and pre-process spPSCs $\bigcirc$ TIMING Approximately $60 \mathrm{~min}$ or more for each cell recorded (depending on the amount of data collected)}

3| If you have acquired data with agonist-evoked responses from outside-out patches, skip Steps 4-16 and continue with Step 17. However, consult Step 9 concerning the possibility of drift in patch responses and Step 12 concerning the possible need for alignment of patch responses.

$4 \mid$ Conditional data conversion. Depending on the software package used to acquire electrophysiological data, you may need to convert your data records into a format that can be read by the software used to detect spPSCs (see below). The Axon Binary Format (ABF) Utility software (Synaptosoft) can be used to convert several different data formats into ABF, which can be read by several software packages.

5| Detect spontaneous synaptic events. Different programs use different algorithms for detecting spPSCs, with each algorithm having strengths and weaknesses. For published methods of event detection, including comparisons of different approaches, see refs. 63-66. Mini Analysis (Synaptosoft) is a good software package for detecting spPSCs and is used by many laboratories. A specific advantage of detecting events with Mini Analysis is that the user can visually verify and edit individual events after detection. A similar approach can be employed with the NeuroMatic software package (see above). This allows for a very flexible workflow where fast, automatic detection can be combined with post hoc, manual editing of individual events by eye.

6| Pre-process detected events by isolating events that are well separated from other events. Although all events have to be detected for a frequency analysis, for example, only events that are temporally well separated from other events can be used for non-stationary noise analysis. Well-separated events can be isolated using an inter-event time interval criterion in such a way that each event contains a sufficient stretch of pre- and post-event baseline free from other spontaneous events.

7| Align the detected events by a temporal criterion (Fig. 1b), for example, by time of steepest rise or by $50 \%$ rise (i.e., the point in time in which the event has reached $50 \%$ of its peak amplitude). The exact criterion is not critical, as the events will be re-aligned later.

8| Export the detected events in a format that can be read by the software for non-stationary noise analysis.

ASCII (American Standard Code for Information and Interchange)-formatted text files can be read by most software packages, but the format is not very compact. The files used for transferring data between programs can be deleted afterward. This step is unnecessary if the same software environment is used for both detection and further non-stationary noise analysis.

\section{Testing detected spPSCs for time stability TIMING Approximately 15-60 min for each cell to be analyzed}

9| The detected spPSCs must first be tested for time stability to ensure that there are no time-dependent changes in the kinetic properties and amplitude of the events ${ }^{2,46,52}$ that might alert one to potential problems with the recording and might otherwise invalidate the non-stationary noise analysis. Such changes could be caused by, say, changes in the series resistance of the recording. Low-pass filter the population of spPSCs at, for example, $2 \mathrm{kHz}$ (digital Gaussian filter) without overwriting the original population of events.

Note that because low-pass filtering can affect the estimates obtained from non-stationary noise analysis, it is desirable to perform the non-stationary noise analysis itself with as little low-pass filtering as possible. For the pre-processing of data, however, it can be unrealistic to analyze waveforms with as little filtering as for the final non-stationary noise analysis. Measure the peak amplitude, $10-90 \%$ (alternatively $20-80 \%$ ) rise time and a decay time course parameter of each event (see Fig. 1c for definitions of these parameters). The decay time course parameter can be either the decay time constant obtained with a mono-exponential curve fit or the half-decay time (i.e., the time interval from the peak amplitude to the half-maximum amplitude during the decay phase). Examples of the distributions of measurements for an experimental recording are illustrated in Figure 1d-f. When testing for time stability, it is crucial that the spPSCs are analyzed in the same order in which they appeared in the original recording.

Slow, approximately linear drift can sometimes be observed in patch recordings-for example, drifts in electrode potentials or rundown of responses caused by washout of cytoplasmic constituents. For responses evoked from an invariant population of ion 

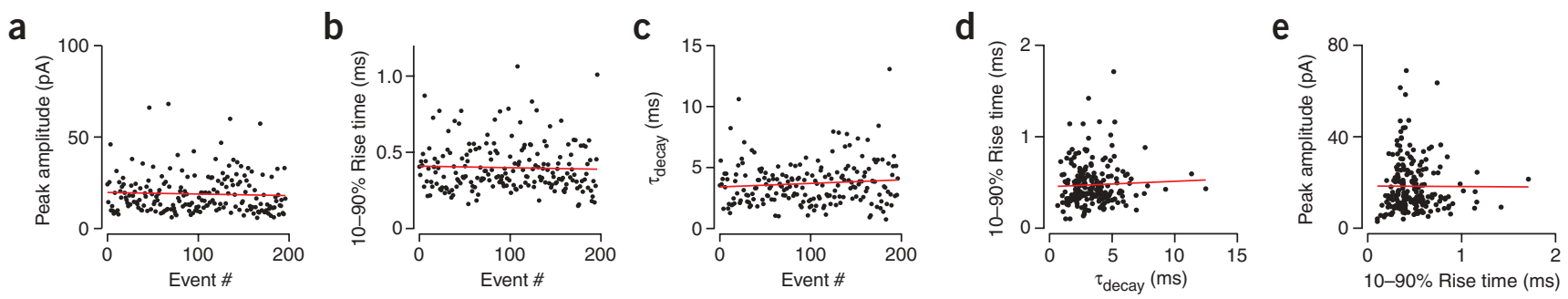

Figure 2 | Example of analysis of time stability and correlation between waveform parameters of glycinergic spontaneous inhibitory postsynaptic currents (spIPSCs) in whole-cell recording from AII amacrine cells in a retinal slice preparation (as in Fig. 1). (a-c) Plots of peak amplitude (a), 10-90\% rise time (b) and $\tau_{\text {decay }}$ (c; single-exponential fit) as a function of event number for consecutive spIPSCs; no time-dependent correlation (red trace in each graph indicates linear fit). For peak amplitude, Spearman's $R=-0.06(P=0.43)$. For $10-90 \%$ rise time, Spearman's $R=-0.02(P=0.81)$. For $\tau_{\text {decay' }}$ Spearman's $R=-0.01(P=0.92)$. (d-f) Relationships between spIPSC waveform parameters (red trace in each graph indicates linear fit): (d) spIPSC $10-90 \%$ rise time versus $\tau_{\text {decay; }}$ (e) spIPSC peak amplitude versus $10-90 \%$ rise time; (f) spIPSC peak amplitude versus $\tau_{\text {decay. }}$. There was no correlation between $10-90 \%$ rise time and $\tau_{\text {decay }}$ (Spearman's $R=0.03 ; P=0.63$ ) or between peak amplitude and $10-90 \%$ rise time (Spearman's $R=0.06 ; P=0.37$ ). There was a significant positive correlation between peak

f

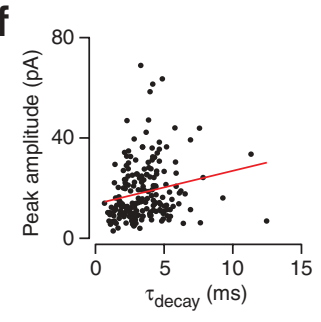
amplitude and $\tau_{\text {decay }}$ (Spearman's $R=0.25 ; P=0.0003$ ), but this is opposite to what is expected if it was due to differential electrotonic filtering ${ }^{26}$. Data in panels a-f modified, with permission, from ref. 26, (C) (2006) The Physiological Society.

channels (e.g., from outside-out patches), it is possible to correct for such drift by calculating the variance from the ensemble average of the squared differences of successive records (see discussion in ref. 67).

10| For each parameter (peak amplitude, rise time and decay time parameter), number each individual measurement according to event number and test for time stability using Spearman's rank-order correlation test ${ }^{46,52}$ (see, e.g., ref. 68 for algorithm). The NeuroMatic software package (see above), contains code for a variable-size sliding-window algorithm. For the complete population of detected events (from a single cell) and for a given parameter, it will return the beginning and end event numbers for the maximum number of consecutive spPSCs that do not display any significant time-dependent correlation $(P>0.05)$. An example of time stability analysis applied to experimental data is illustrated in Figure $2 \mathrm{a}-\mathrm{c}$. An example of IGOR Pro code (function 'Time_Stability') for time stability testing (adapted from NeuroMatic) can be found in the Supplementary Data.

Although using Spearman's rank-order correlation test is explicit with respect to the criteria employed for the test of time stability, the result should be treated with caution if simple visual inspection of the same data reaches the opposite conclusion with respect to the presence or absence of time stability.

\section{Testing detected spPSCs for correlations between waveform parameters TIMING Approximately 10-20 min for each cell to be analyzed}

11 For a population of spPSCs, there is always variability in peak amplitudes, rise times and decay times. This variability is due to a combination of stochastic gating, quantal variability, potential differences in physical and/or electrotonic location of synaptic sites relative to the recording electrode and potential heterogeneity of postsynaptic receptors at different synaptic sites. A series of events displaying time stability (see Step 9) is next tested for correlations between the waveform parameters to evaluate the presence of differential electrotonic filtering. Spearman's rank-order correlation test can also be used to test for correlations between rise time and amplitude, between amplitude and decay time (decay time constant or half-decay time) and between rise time and decay time (decay time constant or half-decay time) ${ }^{46,52}$. The NeuroMatic software package (see above) contains code that can be used for this purpose. An example of IGOR Pro code (function 'Spearman_Rank_Corr') adapted from NeuroMatic can be found in the Supplementary Data. An example of experimental data where the relationships between rise time, peak amplitude and decay time have been analyzed is illustrated in Figure $\mathbf{2 d} \mathbf{d} \mathbf{f}$. The ensemble of consecutive events that displays both time stability and lack of correlations between waveform parameters can now be used for non-stationary noise analysis without the imposed digital Gaussian filter (further analysis at, e.g., $5 \mathrm{kHz}$ ).

\section{Alignment of detected waveforms (spPSCs) $\bigcirc$ TIMING Approximately 5-15 min for each cell to be analyzed}

12| For spPSCs, there are no stimuli that can be used to synchronize or align the waveforms. A recent study compared different methods of aligning simulated data traces (after artificial desynchronization) with respect to obtaining non-stationary noise analysis results as similar as possible to those obtained directly from the original data ${ }^{50}$. Re-alignment by the point of steepest rise of the current trace, as used in the study with peak-scaled non-stationary noise analysis by Traynelis et al. ${ }^{45}$, was found to be the simplest and most effective procedure among those examined. The point of steepest rise corresponds to the location of the minimum value (for inward currents) or maximum value (for outward currents) of the first time derivative of the event waveform. As the first derivative is so noisy, this method normally involves either fitting a function to the rising phase or some sort of 
Figure 3 | Stages in the procedure for conventional non-stationary noise analysis of an ensemble of events without quantal variability. (a) Schematic view of matrix (left) containing digitized data of ensemble of $n$ events $\left(e_{1}, \ldots, e_{n}\right)$, each with duration from $t_{0}$ to $t_{\text {end }}$. Each column contains data for all sample points of a single event and each row contains data for all events at corresponding sample points. Averaging all elements in a single row consecutively for all rows generates the ensemble mean (middle), and calculating the variance for all elements in a single row consecutively for all rows generates the ensemble variance (right). (b) Matrix containing data for $n=1,000$ spontaneous postsynaptic current (spPSC)-like events stochastically simulated as described in ref. 50 (3 mM agonist for $1 \mathrm{~ms}$; arrow indicates time of application). The three-state kinetic scheme $\left(\mathrm{U}_{\overleftarrow{k-1}}^{\stackrel{k_{+1}}{\rightleftarrows}} \mathrm{B} \underset{\alpha}{\stackrel{\beta}{\rightleftarrows}} 0\right)$ had the

following states: $\mathrm{U}=$ unbound state, $\mathrm{B}=$ singly liganded state, $0=$ open state ${ }^{35,88}$. The rate constants $\left(k_{+1}=\right.$ binding; $k_{-1}=$ unbinding; $\beta=$ opening; $\alpha=$ closing) had the following values: $k_{+1}=6 \times 10^{6} \mathrm{M}^{-1} \mathrm{~s}^{-1}, k_{-1}=1,000 \mathrm{~s}^{-1}$, $\alpha=100 \mathrm{~s}^{-1}, \beta=1,000 \mathrm{~s}^{-1}$. The number of receptor channels was set to 50 , the driving force was set to $-60 \mathrm{mV}$, the simulation time step was $10 \mu \mathrm{s}$ and the unitary conductance $(\gamma)$ was $50 \mathrm{pS}$. For clarity, no additional noise was added. Event data organized as in a, with response amplitude coded by color (red corresponds to peak amplitude; purple corresponds to baseline, i.e., zero response level). (c) Ensemble mean (left) and variance (right) waveforms generated from data in panel $\mathbf{b}$ (same time axis as in panel $\mathbf{b}$ ). (d,e) Three individual events from matrix in panel $\mathbf{b}$ (panel $\mathbf{d} ; 1-3$ ) and corresponding difference currents (panel $\mathbf{e} ; 1-3$ ) calculated by subtracting ensemble mean current (c) from each individual event. (f) Plot of ensemble current variance (panel $\mathbf{c}$, right) versus mean current (panel $\mathbf{c}$, left) and versus open probability, generated by plotting corresponding points in time without binning. Time range used for the variance versus mean plot corresponds to data points from the peak of the mean waveform to the end of the decay phase. The data points were fitted with equation (3) (overlaid red trace; single-channel conductance $\gamma=49 \mathrm{pS}, N=52$ channels). Notice the higher density of points for lower than for higher values of $I_{\text {mean }}$.

smoothing. An example of IGOR Pro code (function 'Align_Mat') that uses point of steepest rise for alignment can be found in the Supplementary Data. The code involves the use of a built-in binomial (Gaussian) smoothing algorithm, with the number of smoothing operations set empirically via user-input.

Agonist-evoked responses recorded in outside-out patches will be aligned by the stimulus. However, if the responses have been desynchronized because of drift in the system, it may be necessary to re-align them before further processing.

$\triangle$ CRITICAL STEP Accurate alignment is critical for the following analysis.

\section{? TROUBLESHOOTING}

\section{Non-stationary noise analysis (without binning) TIMING Approximately 10-15 min for each cell to be analyzed}

13| We are now ready to perform non-stationary noise analysis on the final ensemble of responses. In this step, two options are available: non-stationary noise analysis without binning and non-stationary noise analysis with binning. As it is easier to understand, and can serve to illustrate the fundamental principles, we will first apply non-stationary noise analysis without binning. In practice, however, it is generally recommended to employ non-stationary noise analysis with binning (see below). In this first example, we will analyze an ensemble of events with no quantal variability; that is, the number of available ion channels is invariant from one trial to the next (as for responses evoked in outside-out patches).

14| Calculate the ensemble mean response and variance for each point in time for the ensemble of individual responses according to equations (1) and (2). The principle of this procedure is illustrated graphically in Figure $3 a$ and for an ensemble of simulated, PSC-like events in Figure 3b,c. Examples of individual events and their corresponding difference currents are illustrated in Figure 3d,e.

15 For each point in time (indexed by $i)$, plot the ensemble variance $\left(\sigma^{2}\right.$; ordinate) against the mean current $(I$; abscissa), omitting the rising phase of the response (Fig. $\mathbf{3 f}$ ).

16 Fit the data points with equation (3), where $i$ is the single-channel current, $N$ is the available number of channels and $\sigma_{b}{ }^{2}$ is the variance of the background noise. The background noise can be estimated from the pre-response baseline, and the fitted 
Figure 4 | Stages in the binning procedure for non-stationary noise analysis. (a) Plot of ensemble mean current response. The amplitude interval from baseline (0) to peak response has been divided into ten equal amplitude bins (borders between amplitude bins indicated by horizontal broken lines). At the level crossings of the borders between bins with the decaying phase of the ensemble mean waveform, the amplitude bins are translated to the corresponding time bins along the time axis (borders between time bins indicated by vertical broken lines). (b) Schematic view of procedure for averaging of mean and variance values within time bins of varying size. Top array (array $A$ ) contains six bins $\left(b_{1}-b_{6}\right)$, with elements belonging to the same bin demarcated by thicker lines. For simplicity of display, each successive bin contains one more element than the preceding bin. Bottom array (array B) contains six elements $\left(e_{1}-e_{6}\right)$, one for each bin of elements in array $A$. Each element in array $B$ stores the average value of the elements in the corresponding bin in array A. (c) Plot of ensemble current variance versus mean current and versus open probability (same data as in Fig. 3f), but after binning (30 bins). Time range used for the variance versus mean plot corresponds to data points from the peak of the mean waveform to the end of the decay phase. The data points were fitted with equation (3) (overlaid red trace; $\gamma=50 \mathrm{pS}, N=51$ channels).

curve can be constrained for this value. From Figure $\mathbf{3 f}$, it can be seen that the region of the response corresponding to the late decay phase contributes an excess of data points (sampled at constant intervals) because this region spans a long period, but a short range of response values. Thus, this phase of the ensemble mean waveform, containing data points that are not independent of each other, inappropriately is assigned excessive independent weight during curve fitting with equation (3) (ref. 67). This can be avoided with binning as described in the next step.

\section{Non-stationary noise analysis (with binning) TIMING Approximately 10-15 min for each cell to be analyzed}

17| Binning is used to assign similar weights to all phases of the ensemble mean waveform, from the peak to the end of the decay, and accounts at least partially for correlations of current fluctuations within neighboring regions ${ }^{67,69}$. The principle of binning is to divide the mean current amplitude into a number of bins of equal amplitude. For a mean waveform with exponential decay, this will correspond to dividing the mean current decay phase into successive intervals of variable length. Binning is common to both conventional and peak-scaled non-stationary noise analysis. For simplicity, the binning procedure is illustrated step by step for conventional analysis only.

18| At this point, two options are available: (A) conventional non-stationary noise analysis (without peak scaling) to be used for patch responses and for PSCs with no quantal variability and (B) peak-scaled non-stationary noise analysis to be used for events with quantal variability.

\section{(A) Conventional non-stationary noise analysis (without peak scaling)}

(i) Calculate the ensemble mean response and variance for each point in time for the ensemble of individual responses (as in Step 14), according to equations (1) and (2).

(ii) Divide the amplitude interval from the peak to the baseline of the ensemble mean waveform into a number of equal intervals (e.g., 30-100 bins). In this way, each interval (bin) will correspond, on average, to the same number of channel closings $\mathrm{s}^{45,67}$. The procedure is illustrated schematically in Figure 4a.

(iii) Translate the amplitude intervals to the corresponding time intervals for the mean waveform (Fig. 4a). If the ensemble mean was compiled from a large enough number of individual events, the noise will be low and the translation can be performed by calculating the $X$-value (point in time) for each $Y$-value (the amplitude level that separates two bins; Fig. 4a). An example of IGOR Pro code (function 'Find_Levels') for setting the number of bins and detecting the corresponding interval levels along the time axis can be found in the Supplementary Data.

(iv) If the noise is high, employing a level-detection algorithm directly on the mean response will give rise to errors. In this case, an alternative is to use an idealized waveform of the ensemble mean response to translate the amplitude intervals to the corresponding time intervals. The idealized waveform can be obtained by fitting the following function, which combines two exponential functions, to the ensemble mean waveform,

$$
\begin{aligned}
& I(t)=A\left[1-\exp \left(-(t-\delta) / \tau_{\text {rise }}\right)\right] \times \exp \left[-(t-\delta) / \tau_{\text {decay }}\right] \text { for } t \geq \delta \\
& I(t)=0 \text { for } t<\delta,
\end{aligned}
$$


Figure 5 | Stages in the procedure for peak-scaled non-stationary noise analysis (NSNA) of an ensemble of events with quantal variability. Data simulated for spontaneous postsynaptic current (spPSC)-like events as in Figure $3(n=1,000)$, but with quantal variability mimicked by varying the number of available channels randomly between trials (mean $=50$; Gaussian distribution with s.d. $=10$; see ref. 50 for details). (a) Three individual events from the ensemble (1-3). (b,c) The corresponding difference currents calculated without peak scaling (b; conventional non-stationary noise analysis; 1 -3) and with peak scaling (c; peak-scaled non-stationary noise analysis; $1-3$ ) of the ensemble mean waveform before subtracting it from each individual event. (d) Ensemble mean current waveform.

(e) Ensemble variance waveform resulting from conventional non-stationary noise analysis (no peak scaling). (f) Ensemble variance waveform resulting from peak-scaled non-stationary noise analysis. Notice difference in shape and amplitude scaling of variance waveforms in panels $\mathbf{e}$ and $\mathbf{f}$. (g) Plot of ensemble current variance calculated with peak scaling (panel $\mathbf{f}$ ) versus mean current (panel d); 30 bins. The data points were fitted with equation (3) (overlaid red trace; $\gamma=47 \mathrm{pS}, N=41$ channels). (h) Plot of ensemble current variance calculated without peak scaling (panel e) versus mean current (panel d); 30 bins. The initial (leftmost) data points were fitted with a straight line (overlaid red trace; $\gamma=51 \mathrm{pS}$ ). Time range used for the variance versus mean plots corresponds to data points from the peak of the mean waveform to the end of the decay phase (panels $\mathbf{g}, \mathbf{h}$ ).

where $\delta$ is the delay to onset of the response, $A$ describes the peak amplitude and $\tau_{\text {rise }}$ and $\tau_{\text {decay }}$ are the time constants of the rise and decay phases, respectively.

(v) Calculate the average of the ensemble mean current and ensemble variance (each calculated across the individual event waveforms included in the ensemble; Step (i) above) for each bin (time interval; see Fig. 4b for a schematic illustration).

(vi) From the arrays containing the bin averages of mean current and variance, construct the ensemble variance $\left(\sigma^{2}\right.$; ordinate) versus ensemble mean current ( $I$; abscissa) relationship, where each data point represents the average value for each bin (Fig. 4c).

(vii) Fit the data points with equation (3) and obtain estimates for $i$ and $N$ (Fig. 4c). An example of IGOR Pro code (function 'NPS_NSNA_bin') for conventional non-stationary noise analysis with binning can be found in the Supplementary Data. ? TROUBLESHOOTING

(B) Peak-scaled non-stationary noise analysis

(i) In general, peak-scaled non-stationary noise analysis is used for analysis of spPSCs where it is necessary to compensate for the introduced quantal variability when the responses arise from more than a single release site.

(ii) When calculating the difference current for a given individual event (spPSC), multiply the ensemble mean response by the factor needed to scale its peak response to the response value at the corresponding point in time for the given event.

(iii) Calculate the difference current between the spPSC and the peak-scaled ensemble mean response. Figure 5 shows an example with computer-simulated events with quantal variability (Fig. 5a) and difference currents calculated without peak scaling (Fig. 5 b) and with peak scaling (Fig. 5c; see legend for details).

(iv) Repeat (ii) and (iii) for each spPSC in the ensemble to be analyzed.

(v) From the ensemble of difference waveforms, calculate the ensemble variance for each point in time (Fig. 5e,f).

(vi) From the selected number of bins, calculate the corresponding amplitude intervals and translate them to time intervals (as in Steps 18A (ii), (iii); see Fig. 4a).

(vii) Calculate the average of the ensemble mean current and ensemble variance (each calculated across the individual event waveforms included in the ensemble) for each bin (see Fig. 4b).

(viii) From the arrays containing the bin averages of mean current and variance, construct the ensemble variance $\left(\sigma^{2}\right.$; ordinate) versus ensemble mean current ( $I$; abscissa) relationship, where each data point represents the average value for each bin, omitting the rising phase of the response (Fig. $\mathbf{5 g}$ ).

(ix) Fit the data points with equation (3) (Fig. $\mathbf{5 g}$ ). If the variance versus mean relationship is parabolic, (almost) all data points may be used for curve fitting. If the variance versus mean relationship is skewed, the curve fitting should exclude data points from the maximum variance and to the right (e.g., see ref. 50). In either case, curve fitting can reliably estimate $i$, but the estimated $N$ will not correspond to the (average) number of available channels and no 
information on $P_{0 \text {, peak }}$ is available. If the variance versus mean relationship is parabolic, the estimated $N$ will (ideally) correspond to the average number of channels open at the peak. If the relationship is skewed, $N$ will have no physical interpretation. An example of IGOR Pro code (function 'PS_NSNA_bin') for peak-scaled non-stationary noise analysis (with binning) can be found in the Supplementary Data.

If spPSCs (with quantal variability, i.e., arising from more than a single release site) are investigated using conventional non-stationary noise analysis (without peak scaling), it is to be expected that the variance versus mean curves will not display a parabolic shape; instead, they will deviate upward (Fig. $5 \mathbf{h}$; see ref. 50). Estimates for $i$ can still be made by fitting the initial (leftmost) part of the variance versus mean relationship with a straight line, but the curve fitting will use only a few data points and will, therefore, not be very accurate ${ }^{50}$.

? TROUBLESHOOTING

\begin{abstract}
Bootstrap analysis TIMING Approximately 1-2 $\mathrm{h}$ for each cell to be analyzed (depending on the number of synthetic data sets to be generated and analyzed)

19| For optional bootstrap analysis of statistical errors in the best-fit parameters obtained by non-stationary noise analysis of an ensemble with $n$ events $(1, \ldots, n)$, generate $m$ (e.g., 100) random lists, each with $n$ event numbers. Each event number is selected randomly between 1 and $n$ and corresponds to an event in the original ensemble. For $m$ lists of event numbers, each event number occurs a total of $m$ times, but randomly with respect to the individual lists. Each list of numbers can then be used to generate a synthetic, resampled data set with $n$ events, each randomly selected from the original ensemble. A routine implemented in Mathematica for generating lists of randomized event numbers has been published by Roth and Häusser ${ }^{70}$ (their supplementary material) and a corresponding routine implemented in IGOR Pro (function 'Random_Permutation') can be found in the Supplementary Data. When all synthetic data sets have been generated, the same type of non-stationary noise analysis as carried out for the original data set is repeated for each synthetic data set. For a given parameter obtained from the original data set, the analysis of all synthetic data sets will yield a measure of the statistical error.
\end{abstract}

\title{
Covariance analysis TIMING Approximately 1-4 $\mathrm{h}$ for each cell to be analyzed (depending on the number of responses included in the ensemble mean) \\ 20| For this optional analysis, any of the software packages mentioned above can be used to calculate covariance functions for an ensemble of responses (e.g., IGOR Pro, MATLAB, Mathematica and GNU Octave). In addition, the program PulseTools for analysis of electrophysiological signals (from HEKA Elektronik; http://www.heka.com) also has a function for calculating the covariance matrix, but unless one has acquired the data with a program in the HEKA software family, it is difficult to convert the data into a format that can be read by this program.
}

\section{Optional correction for RC filtering $\bigcirc$ TIMING Approximately 10-15 min for each cell to be analyzed}

21 During a whole-cell voltage-clamp recording, the combination of $R_{\mathrm{s}}$ (series resistance) and $C_{\mathrm{m}}$ (cell membrane capacitance) will have the effect of low-pass filtering the recorded current waveforms ${ }^{71}$. With estimates for $R_{\mathrm{s}}$ and $C_{\mathrm{m}}$, it is possible to correct partially for this filtering ${ }^{72}$. Note that this and similar routines do not correct for any electrotonic filtering that may be present in the data. For an example of the application of this procedure to synaptic data without electrotonic filtering, see ref. 73. An example of IGOR Pro code that can be used for this correction can be found at: http://www.mpibpc.gwdg.de/abteilungen/140/ software/index.html (function 'SeriesresistanceComp' in the file 'Proc02_Apr4Web').

\section{TIMING}

For some of the steps listed, the demand for computing power can be considerable (depending on the amount of data processed), and this determines the time required to complete the analysis.

Steps 1 and 2, data acquisition: 6-12 $\mathrm{h}$ for each recording session

Steps 3-8, detect and pre-process spPSCs: 60 min or more for each cell

Steps 9 and 10, testing detected spPSCs for time stability: 15-60 min for each cell to be analyzed

Step 11, testing detected spPSCs for correlations between waveform parameters: 10-20 min for each cell to be analyzed

Step 12, alignment of detected waveforms (spPSCs): 5-15 min for each cell to be analyzed

Steps 17 and 18, non-stationary noise analysis (with binning): 10-15 min for each cell to be analyzed

Step 19, bootstrap analysis: $1-2 \mathrm{~h}$ for each cell to be analyzed (optional)

Step 20, covariance analysis: $1-4 \mathrm{~h}$ for each cell to be analyzed (optional)

Step 21, correction for RC filtering: 10-15 min for each cell to be analyzed (optional)

\section{? TROUBLESHOOTING}

Troubleshooting advice can be found in Table 1. 
TABLE 1 | Troubleshooting table.

\begin{tabular}{llll}
\hline Step & Problem & Possible reason & Solution \\
\hline 12 & $\begin{array}{l}\text { Alignment of spontaneous postsynaptic } \\
\text { currents (spPSCs) for peak-scaled non- } \\
\text { stationary noise analysis does not }\end{array}$ & The first derivative is too noisy & $\begin{array}{l}\text { Increase the degree of smoothing used in } \\
\text { the alignment function }\end{array}$
\end{tabular}

Some events are aligned on a noise peak instead of the peak response

18A Conventional non-stationary noise analysis of an ensemble of outside-out patch responses produces a variance versus mean curve that does not appear parabolic

Peak-scaled non-stationary noise analysis of an ensemble of spPSCs produces a variance versus mean curve that appears flat (instead of parabolic or skewed)

Peak-scaled non-stationary noise analysis of an ensemble of spPSCs produces a variance versus mean curve that appears skewed (instead of parabolic)
The number of responses in the ensemble is not large enough, resulting in too much noise

There was drift in the system during sampling, giving rise to inadequate alignment of the responses

The number of responses in the ensemble is not large enough, resulting in too much noise

The events in the ensemble have not been adequately aligned

The events in the ensemble have not been adequately aligned

A skewed variance versus mean relationship is caused by the kinetic properties of the receptor channels
Eliminate events with sharp noise peaks

The patch has to be eliminated from the analysis

Check alignment of responses carefully; if necessary, attempt re-alignment and examine whether this changes the results

The cell has to be eliminated from the analysis

Check alignment of spPSCs carefully; if necessary, attempt re-alignment and examine whether this changes the results

Check alignment of spPSCs carefully; if necessary, attempt re-alignment and examine whether this changes the results

If receptor channels in outside-out patches from the same cells display similar properties to the synaptic receptor channels, perform peak-scaled non-stationary noise analysis of patch responses to see whether you obtain a skewed variance versus mean relationship here as well. If possible, subject the ensemble of patch responses to covariance analysis and compare the decay time course of the covariance function with that of the macroscopic patch responses

We have included troubleshooting advice only for that part of the protocol that pertains directly to the off-line analysis. Note that the guide illustrates only some of the potential difficulties that can be encountered for a particular type of receptor channel. For troubleshooting advice on data acquisition, see, e.g., refs. 12,22,59-61.

\section{ANTICIPATED RESULTS}

Under stable recording conditions, and with reasonable conditions for voltage and space clamp, the number of spPSCs for a given cell that pass the criteria for time stability and do not display statistically significant correlations between synaptic waveform parameters (indicative of differential electrotonic filtering) can represent up to $95-100 \%$ of the total number of events recorded from a given cell ${ }^{26,52}$. Several studies have employed whole-cell recordings of spPSCs and non-stationary noise analysis (typically peak-scaled) to estimate the single-channel conductance of synaptic receptor channels (e.g., see refs. $26,31,42,45,46,48,52,53,74-77)$. In cases where it has been possible to compare the conductance estimates obtained from non-stationary noise analysis with those obtained from directly resolved single-channel openings in the spPSCs, a reasonably good correspondence between the estimates can be observed ${ }^{26,31}$. On the other hand, in cases where such comparisons are not available, it might be possible to compare the conductance estimates for spPSCs with those obtained from directly resolved single-channel activity and/or non-stationary noise analysis of evoked responses in outside-out patches from the same cell type (e.g., see refs. 52,74,75,78).

For these reasons, we believe that, in general, it is desirable to attempt to obtain both types of data (spPSCs and outside-out patch responses) for a given cell type. However, it cannot be assumed a priori that the receptor channels in patches will necessarily be identical or equivalent to those in the synapses, and there are important caveats that should be borne in mind. 
Patches are by necessity excised from extrasynaptic, as opposed to synaptic, membrane and may therefore have receptor channels with different subunit composition and receptor channels that exist in association with different accessory proteins or cytoskeletal elements. Even in cases where synaptic and extrasynaptic receptor channels are identical, the process of excising outside-out patches might influence or disrupt the interaction of the receptor channels with such accessory proteins and cytoskeletal elements and, in addition, might change the phosphorylation state of the receptor channels. At the same time, it is often exactly such experiments that can be important for assessing the similarity, or lack thereof, between synaptic and extrasynaptic receptor channels.

Another important reason for attempting to combine peak-scaled non-stationary noise analysis of spPSCs with ultrafast application of agonist to outside-out patches (irrespective of the location from which the patches are excised) is the inability of peak-scaled non-stationary noise analysis to yield information on the $P_{\mathrm{o}}$ attained the peak of the average spPSC. Although dividing the peak amplitude of an average PSC by the single-channel current (estimated by peak-scaled non-stationary noise analysis) yields the average number of ion channels open at the peak of the PSC, it does not yield information on the average number of receptor channels exposed to neurotransmitter. However, when an outside-out patch is exposed to brief pulses of neurotransmitter at concentrations in the millimolar range generally considered to be reached in the synaptic cleft ${ }^{13}$, conventional non-stationary noise analysis will yield information not only on the single-channel conductance, but also on

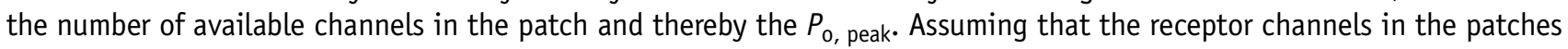
are similar to those mediating the spPSCs, the result of dividing the average number of receptor channels open at the peak of the PSC by the $P_{0 \text {, peak }}$ will equal the average number of receptor channels exposed to neurotransmitter. Such procedures have been used in a number of studies of synaptic transmission in the CNS (e.g., see refs. 26,52,74,75,78).

Peak-scaled non-stationary noise analysis of PSCs has also proven to be a useful tool for investigations of changes in synaptic properties related to development, synaptic plasticity and similar phenomena. In this context, the critical issue has been to decide whether changes in the amplitude of PSCS are caused by a change in the single-channel conductance and/or a change in the number of open channels (e.g., see refs. 6,7,79-86). In this type of investigation, the accuracy of the estimate of the single-channel conductance might be less important than the ability to demonstrate a change in this parameter. Even when the conditions for peak-scaled non-stationary noise analysis are not optimal, the results of a comparison between conditions can provide essential mechanistic information. It is important, however, that there are no other differences between the conditions being compared, such as systematic differences in series resistance and/or electrotonic filtering, that can cause the observed effects or lack thereof.

Similar considerations can be important for experimental controls in work with non-stationary noise analysis. Such controls might employ manipulations that would be expected to alter (or not to alter) one or other of the deduced parameters, with a view to assessing the ability to detect relevant changes. For example, analyzing recordings in the presence of low concentrations of competitive, slowly dissociating antagonists would not be expected to reduce the estimate of $i$ (obtained by conventional or peak-scaled non-stationary noise analysis), but should reduce the estimate of $N$ (obtained by conventional non-stationary noise analysis for patch responses). In the same way, analyzing recordings (patch responses or spPSCs) obtained at different holding potentials would not be expected to change the estimate of $i$. Finally, analyzing recordings obtained in the presence of an allosteric modulator might be expected to change the peak open probability without changing the estimates for $i$ or $N$. The details of such controls will depend on the specific properties of the receptor channels investigated.

Although ultrafast application of agonist to outside-out patches is often used to study kinetic properties of receptor channels, such studies have also employed non-stationary noise analysis to study neuromodulatory changes of receptor channels that can be difficult to resolve with single-channel recording. For example, non-stationary noise analysis has been used to distinguish between changes in $P_{0 \text {, peak }}$ and single-channel conductance in studies of the modulatory control of glutamate receptors by cAMP-dependent protein kinase ${ }^{87}$.

Note: Supplementary information is available via the HTML version of this article.

ACKNOWLEDGMENTS Financial support from the Norwegian Research Council (NFR 155397/310 and 161217/V40) and the Faculty of Medicine at the University of Bergen (fellowship for M.L.V.) is gratefully acknowledged.

COMPETING INTERESTS STATEMENT The authors declare that they have no competing financial interests.

Published online at http://www.natureprotocols.com

Reprints and permissions information is available online at http://npg.nature.com/ reprintsandpermissions

1. Eccles, J.C. The Physiology of Synapses (Springer, Berlin, Heidelberg, New York, 1964).
2. Silver, R.A. \& Farrant, M. Neurotransmitter-gated ion channels in dendrites. in Dendrites (eds. Stuart, G., Spruston, N. \& Häusser, M.) 114-138 (0xford University Press, 0xford, New York, 1999).

3. Jonas, P., Major, G. \& Sakmann, B. Quantal components of unitary EPSCs at the mossy fibre synapse on CA3 pyramidal cells of rat hippocampus. J. Physiol. 472, 615-663 (1993).

4. Spruston, N., Jaffe, D.B., Williams, S.H. \& Johnston, D. Voltage- and space-clamp errors associated with the measurement of electrotonically remote synaptic events. J. Neurophysiol. 70, 781-802 (1993).

5. Spruston, N., Jaffe, D.B. \& Johnston, D. Dendritic attenuation of synaptic potentials and currents: the role of passive membrane properties. Trends Neurosci. 17, 161-166 (1994).

6. Benke, T.A. et al. Mathematical modelling of non-stationary fluctuation analysis for studying channel properties of synaptic AMPA receptors. J. Physiol. 537, 407-420 (2001). 
7. Benke, T.A., Lüthi, A., Isaac, J.T. \& Collingridge, G.L. Modulation of AMPA receptor unitary conductance by synaptic activity. Nature 393, 793-797 (1998).

8. Magee, J.C. \& Cook, E.P. Somatic EPSP amplitude is independent of synapse location in hippocampal pyramidal neurons. Nat. Neurosci. 3, 895-903 (2000).

9. Palmer, M.J. Functional segregation of synaptic $G_{A B A}$ and $G A B A_{C}$ receptors in goldfish bipolar cell terminals. J. Physiol. 577, 45-53 (2006).

10. Oltedal, L., Mørkve, S.H., Veruki, M. \& Hartveit, E. Patch clamp investigations and compartmental modeling of rod bipolar axon terminals in an in vitro thin slice preparation of the mammalian retina. J. Neurophysiol. 97, 1171-1187 (2007).

11. Veruki, M.L., Mørkve, S.H. \& Hartveit, E. Activation of a presynaptic glutamate transporter regulates transmission through electrical signaling. Nat. Neurosci. $\mathbf{9}$ 1388-1396 (2006).

12. Jonas, P. Fast application of agonists to isolated membrane patches. In SingleChannel Recording 2nd edn. (eds. Sakmann, B. \& Neher, E.) 231-243 (Plenum Press, New York, 1995).

13. Clements, J.D. Transmitter timecourse in the synaptic cleft: its role in central synaptic function. Trends Neurosci. 19, 163-171 (1996).

14. Lester, R.A., Clements, J.D., Westbrook, G.L. \& Jahr, C.E. Channel kinetics determine the time course of NMDA receptor-mediated synaptic currents. Nature 346, 565-567 (1990).

15. Hestrin, S. Activation and desensitization of glutamate-activated channels mediating fast excitatory synaptic currents in the visual cortex. Neuron $\mathbf{9}$, 991-999 (1992).

16. Jonas, P. \& Sakmann, B. Glutamate receptor channels in isolated patches from CA1 and CA3 pyramidal cells of rat hippocampal slices. J. Physiol. 455, 143-171 (1992).

17. Raman, I.M. \& Trussell, L.O. The kinetics of the response to glutamate and kainate in neurons of the avian cochlear nucleus. Neuron 9, 173-186 (1992).

18. Spruston, N., Jonas, P. \& Sakmann, B. Dendritic glutamate receptor channels in rat hippocampal CA3 and CA1 pyramidal neurons. J. Physiol. 482, 325-352 (1995).

19. Häusser, M. \& Roth, A. Dendritic and somatic glutamate receptor channels in rat cerebellar Purkinje cells. J. Physiol. 501, 77-95 (1997).

20. Andrásfalvy, B.K. \& Magee, J.C. Distance-dependent increase in AMPA receptor number in the dendrites of adult hippocampal CA1 pyramidal neurons. J. Neurosci. 21, 9151-9159 (2001).

21. Faber, D.S., Korn, H., Redman, S.J., Thompson, S.M. \& Altman, J.S. (eds.) Central Synapses: Quantal Mechanisms and Plasticity (Human Frontier Science Program, Strasbourg, 1998).

22. Trussell, L.O. Recording and analyzing synaptic currents and synaptic potentials. in Current Protocols in Neuroscience 6.10.1-6.10.12 (Wiley, New York, 1999).

23. Takahashi, T. \& Momiyama, A. Single-channel currents underlying glycinergic inhibitory postsynaptic responses in spinal neurons. Neuron 7, 965-969 (1991).

24. Dieudonné, S. Glycinergic synaptic currents in Golgi cells of the rat cerebellum. Proc. Natl. Acad. Sci. USA 92, 1441-1445 (1995).

25. Protti, D.A., Gerschenfeld, H.M. \& Llano, I. GABAergic and glycinergic IPSCs in ganglion cells of rat retinal slices. J. Neurosci. 17, 6075-6085 (1997).

26. Gill, S.B., Veruki, M.L. \& Hartveit, E. Functional properties of spontaneous IPSCs and glycine receptors in rod amacrine (AII) cells in the rat retina. J. Physiol. 575, 739-759 (2006)

27. Robinson, H.P., Sahara, Y. \& Kawai, N. Nonstationary fluctuation analysis and direct resolution of single channel currents at postsynaptic sites. Biophys. J. 59, 295-304 (1991).

28. Silver, R.A., Traynelis, S.F. \& Cull-Candy, S.G. Rapid-time-course miniature and evoked excitatory currents at cerebellar synapses in situ. Nature 355, 163-166 (1992).

29. Clark, B.A., Farrant, M. \& Cull-Candy, S.G. A direct comparison of the singlechannel properties of synaptic and extrasynaptic NMDA receptors. J. Neurosci. 17 107-116 (1997).

30. Billups, D., Liu, Y.B., Birnstiel, S. \& Slater, N.T. NMDA receptor-mediated currents in rat cerebellar granule and unipolar brush cells. J. Neurophysiol. 87, 1948-1959 (2002).

31. Brickley, S.G., Cull-Candy, S.G. \& Farrant, M. Single-channel properties of synaptic and extrasynaptic $G_{A B A_{A}}$ receptors suggest differential targeting of receptor subtypes. J. Neurosci. 19, 2960-2973 (1999).

32. Béhé, P., Colquhoun, D. \& Wyllie, D.J.A. Activation of single AMPA- and NMDAtype glutamate-receptor channels. In Handbook of Experimental Pharmacology: Ionotropic Glutamate Receptors in the CNS Vol. 141 (eds. Jonas, P. \& Monyer, H.) 175-218 (Springer Verlag, Berlin, Heidelberg, 1999).

33. Hamill, O.P., Marty, A., Neher, E., Sakmann, B. \& Sigworth, F.J. Improved patchclamp techniques for high-resolution current recording from cells and cell-free membrane patches. Pflügers Arch. 391, 85-100 (1981).

34. Sakmann, B. \& Neher, E. Single-Channel Recording 2nd edn. (Plenum Press, New York, London, 1995).
35. Colquhoun, D. \& Hawkes, A.G. Relaxations and fluctuations of membrane currents that flow through drug-operated channels. Proc. R. Soc. Lond. B Biol. Sci. 199, 231-262 (1977).

36. Neher, E. \& Stevens, C.F. Conductance fluctuations and ionic pores in membranes. Annu. Rev. Biophys. Bioeng. 6, 345-381 (1977).

37. DeFelice, L.J. Introduction to Membrane Noise (Plenum Press, New York, 1981).

38. Sigworth, F.J. The variance of sodium current fluctuations at the node of Ranvier. J. Physiol. 307, 97-129 (1980).

39. Sigworth, F.J. Covariance of nonstationary sodium current fluctuations at the node of Ranvier. Biophys. J. 34, 111-133 (1981).

40. Sigworth, F.J. Nonstationary noise analysis of membrane currents. In Membranes, Channels, and Noise (eds. Eisenberg, R.S., Frank, M. \& Stevens, C.F.) 21-48 (Plenum Press, New York, 1984).

41. Smith, M.A., Ellis-Davies, G.C. \& Magee, J.C. Mechanism of the distancedependent scaling of Schaffer collateral synapses in rat CA1 pyramidal neurons. J. Physiol. 548, 245-258 (2003).

42. Auger, C. \& Marty, A. Heterogeneity of functional synaptic parameters among single release sites. Neuron 19, 139-150 (1997).

43. Dalby, N.0. \& Mody, I. Activation of NMDA receptors in rat dentate gyrus granule cells by spontaneous and evoked transmitter release. J. Neurophysiol. 90, 786-797 (2003).

44. Dempster, J. Computer Analysis of Electrophysiological Signals (Academic Press, London, 1993).

45. Traynelis, S.F., Silver, R.A. \& Cull-Candy, S.G. Estimated conductance of glutamate receptor channels activated during EPSCs at the cerebellar mossy fiber-granule cell synapse. Neuron 11, 279-289 (1993).

46. Silver, R.A., Cull-Candy, S.G. \& Takahashi, T. Non-NMDA glutamate receptor occupancy and open probability at a rat cerebellar synapse with single and multiple release sites. J. Physiol. 494, 231-250 (1996).

47. Traynelis, S.F. \& Jaramillo, F. Getting the most out of noise in the central nervous system. Trends Neurosci. 21, 137-145 (1998).

48. De Koninck, Y. \& Mody, I. Noise analysis of miniature IPSCs in adult rat brain slices: properties and modulation of synaptic $\mathrm{GABA}_{A}$ receptor channels. J. Neurophysiol. 71, 1318-1335 (1994).

49. Cull-Candy, S.G., Howe, J.R. \& Ogden, D.C. Noise and single channels activated by excitatory amino acids in rat cerebellar granule neurones. J. Physiol. 400, 189-222 (1988).

50. Hartveit, E. \& Veruki, M.L. Studying properties of neurotransmitter receptors by non-stationary noise analysis of spontaneous synaptic currents. J. Physiol. 574, 751-785 (2006).

51. Nusser, Z., Naylor, D. \& Mody, I. Synapse-specific contribution of the variation of transmitter concentration to the decay of inhibitory postsynaptic currents. Biophys. J. 80, 1251-1261 (2001).

52. Momiyama, A. et al. The density of AMPA receptors activated by a transmitter quantum at the climbing fibre-Purkinje cell synapse in immature rats. J. Physiol. 549, 75-92 (2003).

53. Markova, 0. et al. Applicability of peak-scaled nonstationary fluctuation analysis to the study of inhibitory synaptic transmission in hippocampal cultures. Neurophysiology 37, 333-343 (2005).

54. Bendat, J.S. \& Piersol, A.G. Random Data. Analysis and Measurement Procedures 3rd edn. (John Wiley \& Sons, New York, 2000).

55. Celentano, J.J. \& Hawkes, A.G. Use of the covariance matrix in directly fitting kinetic parameters: application to $\mathrm{GABA}_{A}$ receptors. Biophys. J. 87, 276-294 (2004).

56. Efron, B. \& Tibshirani, R. An Introduction to the Bootstrap (Chapman \& Hall/CRC, Boca Raton, 1993).

57. Penner, R. A practical guide to patch clamping. In Single-Channel Recording 2nd edn. (eds. Sakmann B. \& Neher, E.) 3-30 (Plenum Press, New York, 1995).

58. Sakmann, B. \& Stuart, G. Patch-pipette recordings from the soma, dendrites, and axons of neurons in brain slices. In Single-Channel Recording 2nd edn. (eds. Sakmann, B. \& Neher, E.) 199-211 (Plenum Press, New York, 1995).

59. Bischofberger, J., Engel, D., Li, L., Geiger, J.R.P. \& Jonas, P. Patch-clamp recording from mossy fiber terminals in hippocampal slices. Nat. Protoc. 1, 2075-2081 (2006).

60. Castañeda-Castellanos, D.R., Flint, A.C. \& Kriegstein, A.R. Blind patch clamp recordings in embryonic and adult mammalian brain slices. Nat. Protoc. 1, 532-542 (2006).

61. Davie, J.T. et al. Dendritic patch-clamp recording. Nat. Protoc. 1, 1235-1247 (2006).

62. Silberberg, S.D. \& Magleby, K.L. Preventing errors when estimating single channel properties from the analysis of current fluctuations. Biophys. J. 65, 1570-1584 (1993).

63. Ankri, N., Legendre, P., Faber, D.S. \& Korn, H. Automatic detection of spontaneous synaptic responses in central neurons. J. Neurosci. Methods 52, 87-100 (1994). 
64. Clements, J.D. \& Bekkers, J.M. Detection of spontaneous synaptic events with an optimally scaled template. Biophys. J. 73, 220-229 (1997).

65. Hwang, T.N. \& Copenhagen, D.R. Automatic detection, characterization, and discrimination of kinetically distinct spontaneous synaptic events. J. Neurosci. Methods 92, 65-73 (1999).

66. Kudoh, S.N. \& Taguchi, T. A simple exploratory algorithm for the accurate and fast detection of spontaneous synaptic events. Biosens. Bioelectron. 17, 773-782 (2002).

67. Heinemann, S.H. \& Conti, F. Nonstationary noise analysis and application to patch clamp recordings. Methods Enzymol. 207, 131-148 (1992).

68. Press, W.H., Teukolsky, S.A., Vetterling, W.T. \& Flannery, B.P. Numerical Recipes in C. The Art of Scientific Computing 2nd edn. (Cambridge University Press, Cambridge, UK, 1992).

69. Steffan, R. \& Heinemann, S.H. Error estimates for results of nonstationary noise analysis derived with linear least squares methods. J. Neurosci. Methods $\mathbf{7 8}$, 51-63 (1997).

70. Roth, A. \& Häusser, M. Compartmental models of rat cerebellar Purkinje cells based on simultaneous somatic and dendritic patch-clamp recordings. J. Physiol 535, 445-472 (2001).

71. Marty, A. \& Neher, E. Tight-seal whole-cell recording. in Single-Channel Recording 2nd edn. (eds. Sakmann, B. \& Neher, E.) 31-52 (Plenum Press, New York, 1995).

72. Traynelis, S.F. Software-based correction of single compartment series resistance errors. J. Neurosci. Methods 86, 25-34 (1998).

73. Neher, E. \& Sakaba, T. Estimating transmitter release rates from postsynaptic current fluctuations. J. Neurosci. 21, 9638-9654 (2001).

74. Perrais, D. \& Ropert, N. Effect of zolpidem on miniature IPSCs and occupancy of postsynaptic $\mathrm{GABA}_{\mathrm{A}}$ receptors in central synapses. J. Neurosci. 19, 578-588 (1999).

75. Singer, J.H. \& Berger, A.J. Contribution of single-channel properties to the time course and amplitude variance of quantal glycine currents recorded in rat motoneurons. J. Neurophysiol. 81, 1608-1616 (1999).

76. Stern, J.E., Galarreta, M., Foehring, R.C., Hestrin, S. \& Armstrong, W.E. Differences in the properties of ionotropic glutamate synaptic currents in oxytocin and vasopressin neuroendocrine neurons. J. Neurosci. 19, 3367-3375 (1999).

77. Sahara, Y. \& Takahashi, T. Quantal components of the excitatory postsynaptic currents at a rat central auditory synapse. J. Physiol. 536, 189-197 (2001).
78. Nusser, Z., Cull-Candy, S. \& Farrant, M. Differences in synaptic GABA $A_{A}$ receptor number underlie variation in GABA mini amplitude. Neuron 19, 697-709 (1997).

79. Otis, T.S., De Koninck, Y. \& Mody, I. Lasting potentiation of inhibition is associated with an increased number of $\gamma$-aminobutyric acid type A receptors activated during miniature inhibitory postsynaptic currents. Proc. Natl. Acad. Sci. USA 91, 7698-7702 (1994).

80. Cohen, A.S., Lin, D.D. \& Coulter, D.A. Protracted postnatal development of inhibitory synaptic transmission in rat hippocampal area CA1 neurons. J. Neurophysiol. 84, 2465-2476 (2000).

81. Kilman, V., van Rossum, M.C.W. \& Turrigiano, G.G. Activity deprivation reduces miniature IPSC amplitude by decreasing the number of postsynaptic GABA receptors clustered at neocortical synapses. J. Neurosci. 22, 1328-1337 (2002).

82. Leao, R.N. et al. Differences in glycinergic mIPSCs in the auditory brain stem of normal and congenitally deaf neonatal mice. J. Neurophysiol. 91, 1006-1012 (2004).

83. Linnemann, C., Sultan, F., Pedroarena, C.M., Schwarz, C. \& Thier, P. Lurcher mice exhibit potentiation of $\mathrm{GABA}_{\mathrm{A}}$-receptor-mediated conductance in cerebellar nuclei neurons in close temporal relationship to Purkinje cell death. J. Neurophysiol. 91, 1102-1107 (2004).

84. Baldelli, P., Hernandez-Guijo, J.-M., Carabelli, V. \& Carbone, E. Brain-derived neurotrophic factor enhances GABA release probability and nonuniform distribution of $\mathrm{N}$ - and $\mathrm{P} / \mathrm{Q}$-type channels on release sites of hippocampal inhibitory synapses. J. Neurosci. 25, 3358-3368 (2005).

85. Gordon, G.R. et al. Norepinephrine triggers release of glial ATP to increase postsynaptic efficacy. Nat. Neurosci. 8, 1078-1086 (2005).

86. Ling, D.S., Benardo, L.S. \& Sacktor, T.C. Protein kinase Mร enhances excitatory synaptic transmission by increasing the number of active postsynaptic AMPA receptors. Hippocampus 16, 443-452 (2006).

87. Banke, T.G. et al. Control of GluR1 AMPA receptor function by cAMP-dependent protein kinase. J. Neurosci. 20, 89-102 (2000).

88. del Castillo, J. \& Katz, B. Interaction at end-plate receptors between different choline derivatives. Proc. R. Soc. Lond. B Biol. Sci. 146, 369-381 (1957). 